\title{
Synthesis of Tin Oxide Thick Film and Its Investigation as a LPG Sensor at Room Temperature
}

\author{
Tripti Shukla \\ Department of Physics, Sant Gadge Baba Amravati University, Amravati, India \\ Email: triptishukla.20@rediffmail.com
}

Received May 4, 2012; revised June 5, 2012; accepted July 7, 2012

\begin{abstract}
Present paper reports the synthesis of $\mathrm{SnO}_{2}$, its characterization and performance as Liquefied Petroleum Gas (LPG) Sensor. XRD pattern revealed the tetragonal crystalline nature of the material. Crystallites sizes were in the range 14 $30 \mathrm{~nm}$. Tin oxide thick film was prepared by using screen printing technique. After that these were investigated through SEM. SEM image of thick-film surface was spherical in shape and porous. Further at room temperature, the film was exposed to LPG in a controlled gas chamber and variations in resistance with the concentrations of LPG were observed. The maximum value of average sensitivity of thick film was $37 \mathrm{M} \Omega / \mathrm{min}$ for 5 vol. \% of LPG. Sensor responses as a function of exposure and response times were also estimated and maximum sensor response were found 273 and 312 for 4 and 5 vol\% of LPG respectively.
\end{abstract}

Keywords: LPG Sensor; Thick Film; Sensitivity; SEM; XRD

\section{Introduction}

The properties of metal oxides have received a great deal of interest for many years, due to applications in various fields such as solar cells, optical devices and oxidation catalysts. Numerous metal oxide semiconductor materials have been reported to be usable as gas sensor, such as $\mathrm{ZnO}, \mathrm{SnO}_{2}$, and $\mathrm{TiO}_{2}$ and so on [1-10]. These materials have non-stoichiometric structure, so free electron, originating from oxygen vacancies contribute to electrical conductivity. Tin oxide $\left(\mathrm{SnO}_{2}\right)$ is extensively studied because of its interest in both the application and the fundamental research in the last decade due to its remarkable optical and electrical properties. Tin oxide is a wide band gap $(3.6 \mathrm{eV})$ having its conductivity depending upon oxygen vacancies that act as donors [11-14].

Several deposition techniques have been developed to grow undoped and doped $\mathrm{SnO}_{2}$ films such as spray pyrolysis evaporation, chemical vapor deposition, sol gel technique, magnetron sputtering pulsed laser deposition and screen printing technique. The production of sensing layers by screen-printing technology has met the great interest in this field. In fact, screen-printing is a simple and automated manufacturing technique that allows the production of low cost and robust chemical sensors with good reproducibility. Such technique allows the deposition of a controlled amount of paste. Thick films are suitable for such sensors since the gas sensing properties are related to the material surface and the gases are always adsorbed and react with the films surface [15-19]. The development of gas sensors to monitor combustible gases is imperative due to the concern for safety requirements in homes and industries. The liquefied petroleum gas (LPG) is one of extensively used but potentially hazardous gases and its detection is particularly important, because explosion accident may be caused when it leaks out accidentally or by mistake. So the detection of LPG in domestic appliances must be no false or missing alarms during cooking, which requires the equipment to identify LPG [20].

The gas sensing properties of $\mathrm{SnO}_{2}$ semiconductor thick film have been found to depend strongly on the method of processing $[21,22]$. Among the structural parameters, the crystallite size has prominent effect on the gas sensitivity. It is now recognized that $\mathrm{SnO}_{2}$ semiconductor thick film can have maximum gas sensitivity only if the nanocrystallite size within the film is comparable with its space-charge layer thickness $[23,24]$. Hence, the major objectives of the present investigation are set to synthesize $\mathrm{SnO}_{2}$ semiconductor thick film, having nanosized crystallites using the screen printing technique. Various analytical techniques such as Scanning Electron Microscopy (SEM), X-ray Diffraction (XRD) and UVvisible absorption analysis (UV-vis) are utilized in the present investigation for determining the surface morphology, crystallite size and band gap within the film. Besides, the present study also focuses on demonstrating the suitability of such film for sensing LPG gas at room temperature. 


\section{Experimental Procedure}

Tin oxide was prepared by chemical co-precipitation method. $12 \mathrm{~g}$ stannous chloride $\left[\mathrm{SnCl}_{2} \cdot 2 \mathrm{H}_{2} \mathrm{O}\right]$ was dissolved in $95 \mathrm{~mL}$ of isopropyl alcohol to make $1 \mathrm{M}$ solution. Ammonium hydroxide was added drop wise to $1 \mathrm{M}$ stannous chloride dehydrate solution. After vigorous stirring for $6 \mathrm{~h}$, a dispersed solution was formed. This dispersed solution was sonicated for 30 minutes, yielding a precipitate. The precipitated compound was thoroughly washed with distilled water to remove chloride ions. The paste thus formed was screen printed onto an ultrasonically cleaned alumina substrate. Then the thick film was allowed to stabilize at room temperature for $6 \mathrm{~h}$ and calcined at $450^{\circ} \mathrm{C}$ for $1 \mathrm{~h}$. The silver contacts on both the ends of film were made for signal registration. It was exposed to LPG in a self-designed conventional chamber and corresponding variations in resistances with the time were recorded by using a digital millimeter.

\section{Characterization Technique}

\subsection{Scanning Electron Microscopy}

Surface morphology of the sensing element was analyzed using Scanning Electron Microscope unit. SEM image is shown in Figure 1(a). It exhibits that the grown samples consist of spherical grains having more space as pores. Each grain is uniformly distributed over the surface. Figure 1(b) shows the $\mathrm{SEM}$ of $\mathrm{SnO}_{2}$ after exposition of the LPG. These adsorption sites are disappeared after the adsorption of LPG on the surface of the sensing film. The grains were seemed to be swallowed and as a result their sizes became larger.

\subsection{X-Ray Diffraction}

The crystal structure and phase of the powdered sample were analyzed by using X-ray Diffractometer with $\mathrm{Cu}-\mathrm{K}_{\alpha}$ radiation as source having wavelength $1.546 \AA$. Crystal- lite size was estimated using the broadening of XRD peaks by the Debye-Scherer formula which is as follows:

$$
D=\frac{K \lambda}{\beta \cos \theta}
$$

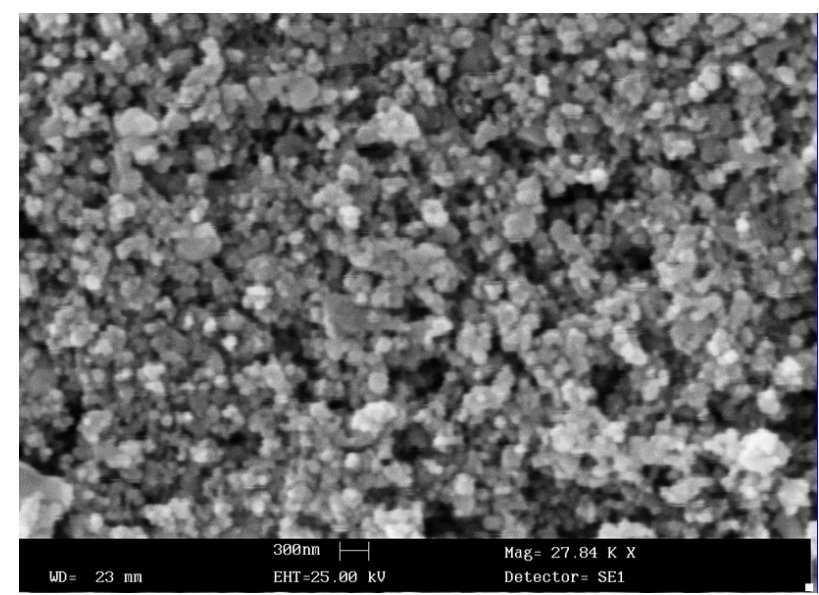

(a)

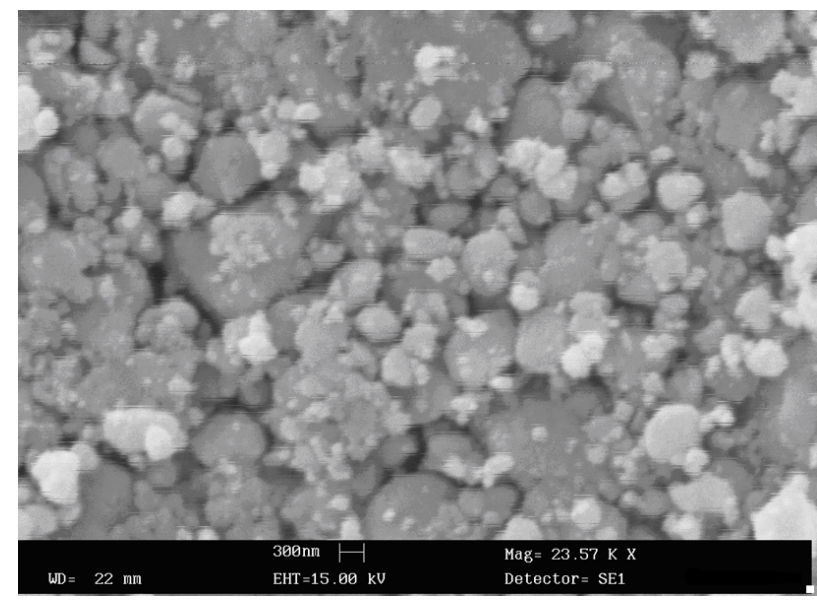

(b)

Figure 1. (a) SEM of $\mathrm{SnO}_{2}$ thick film before exposing LPG; (b) $\mathrm{SEM} \mathrm{of} \mathrm{SnO}_{2}$ thick film after exposing LPG.

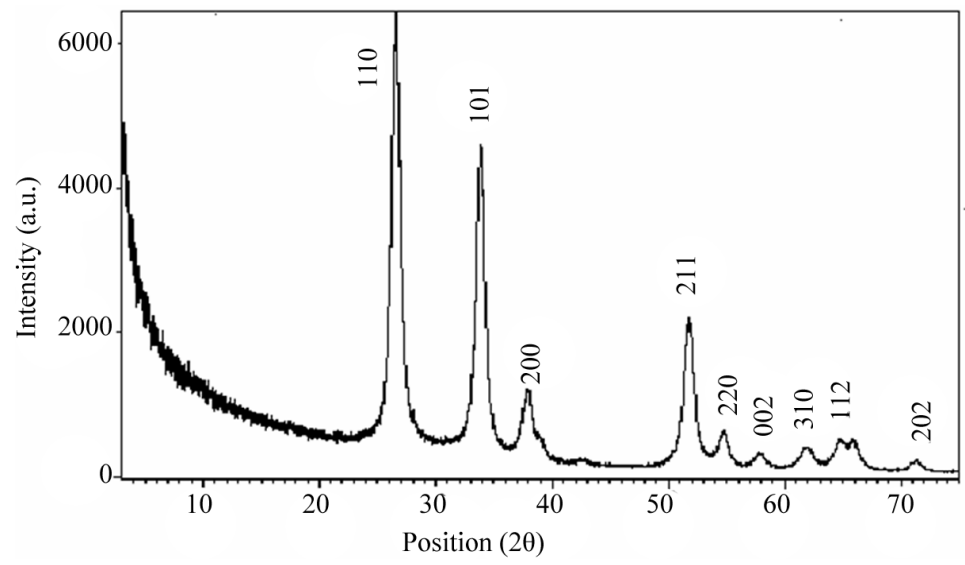

Figure 2. X-ray Diffraction pattern of $\mathrm{SnO}_{2}$ sensing material. 
where $\beta$ is the full width at half maximum (FWHM) of the peak, $\lambda$ is $\mathrm{X}$-ray wavelength, $\theta$ is the Bragg angle and $K=0.94$, a dimensionless constant. Figure 2 shows the XRD pattern of $\mathrm{SnO}_{2}$ powder and confirms the structure. The high intensity peak, centered at $2 \theta=26^{\circ}$ is assigned to tetragonal crystalline $\mathrm{SnO}_{2}$ (110) reflection having'd' spacing $3.35454 \AA$ and FWHM $0.5353^{\circ}$. Also the peak with low intensity at $2 \theta=71^{\circ}$ is assigned to $\mathrm{SnO}_{2}$ (202) reflection having " $d$ " spacing and FWHM 1.31985 $\AA$ and $0.6912^{\circ}$ respectively. Other higher angle reflection such as (101), (200), (211) were indicating tetragonal crystalline nature of $\mathrm{SnO}_{2}$. The crystallite size is calculated by using Debye-Scherrer formula. The minimum crystallite size was found to be $14 \mathrm{~nm}$ at $2 \theta=42.67^{\circ}$ with "d" spacing $2.118945 \AA$ and FWHM $0.7557^{\circ}$.

\subsection{UV-Visible Absorption Analysis}

Optical characterization of the sensing element was done by using UV-visible spectrophotometer (Varian, Carry50Bio). Figure 3 shows UV-visible absorption spectra of tin oxide in UV and visible range. Tin oxide nanoparticles reveal a strong change of their optical absorption when their size is reduced. Therefore, absorption spectra of tin oxide nanoparticles obtained in the UV-visible region show blue shift in the absorption edge at $268 \mathrm{~nm}$ as compared to bulk. The corresponding band gap was found $4.66 \mathrm{eV}$ respectively. It is evident that tin oxide shows significant blue shift of the absorption peak relative to the bulk absorption. This blue shift is useful for gas sensing applications.

\section{Device Assembly}

The main goal of this investigation is to develop a high sensitive LPG sensor and at the same time to analyze the effect of parameters on the gas sensing properties of the thick film. The purpose is not only to evaluate the gas sensing response, but also to reproduce as much as possible a real environment for a working LPG sensor. Therefore, following protocol is used to test the gas sensing behavior of the thick film. The schematic diagram of ex-

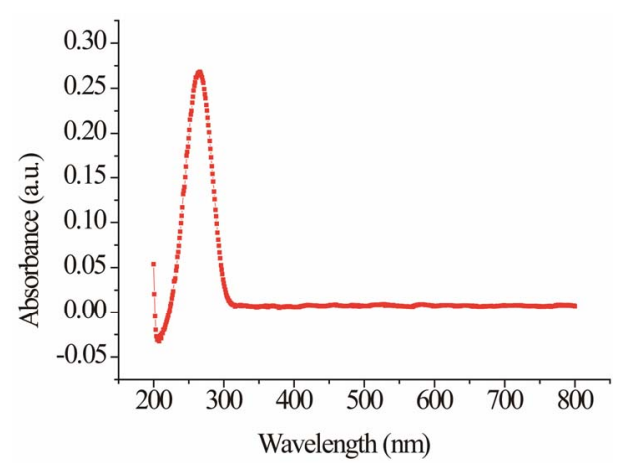

Figure 3. UV-visible spectra of tin oxide. perimental setup is shown in Figure 4(a). The heart part of the device is a resistance measuring thick film holder. It is well fitted in a glass chamber having inlet and outlet knobs for LPG. Inlet knob is associated with the concentration measuring system along with a thermocouple.

\section{Concentration Measuring System}

Figure 4(b) consists of a glass bottle containing double distilled water, which is saturated with LPG, in order to avoid the possibility of dissolution of inserted gas above this bottle, the measuring tube (pipette) is connected by vacuum seal. The cock I is connected to the LPG cylinder and cock II is connected to the inlet of the gas chamber. When the cock I is opened, the LPG from the cylinder is filled in the glass bottle and an equivalent amount of water is displaced in the measuring pipette. When the cock II is opened, a desired amount of gas e.g. 1, 2, 3 vol\% and onwards is cast out in the gas chamber.

\section{Gas Sensing Properties}

The sensitivity of sensing element is defined as the slope of the resistance-time curve and is given below [25]:

$$
S=\Delta R / \Delta t
$$

The sensitivity of the sensor is to be high due to adequate change in resistance $(\Delta R)$ in a minute time $(\Delta t)$ in the presence of LPG, and for an improved performance it is desired that both the changes occur accordingly.

Percentage sensor response for the sensing material is defined as [22]:

$$
\% \text { S.R. }=\frac{\left|R_{a}-R_{g}\right|}{R_{a}} \times 100
$$

where $R_{a}$ and $R_{g}$ are the resistance values of the sensor in air and gas-air mixture, respectively

\section{Results and Discussion}

Prima-facie before the exposition of LPG to the sensing element, the gas chamber was allowed to evacuate at room temperature for $15-20 \mathrm{~min}$ and the stabilized resistance was taken as $R_{a}$. Variations in resistance with the exposure time for different concentrations of LPG were recorded as shown in Figure 5. The resistance of tin oxide thick film increases from initial value with time after exposure after that it becomes constant. Finally the outlet of the chamber was opened, the resistance approaches to their initial value of stabilized resistance in air $\left(R_{a}\right)$ for further range of time. Figure 6 exhibits the variation of average sensitivity with increasing concentration of LPG. The maximum average sensitivity was $37 \mathrm{M} \Omega / \min$ for 5 vol\% of LPG. Small particle size and large specific area contribute to oxygen and reducing gas adsorption on the 


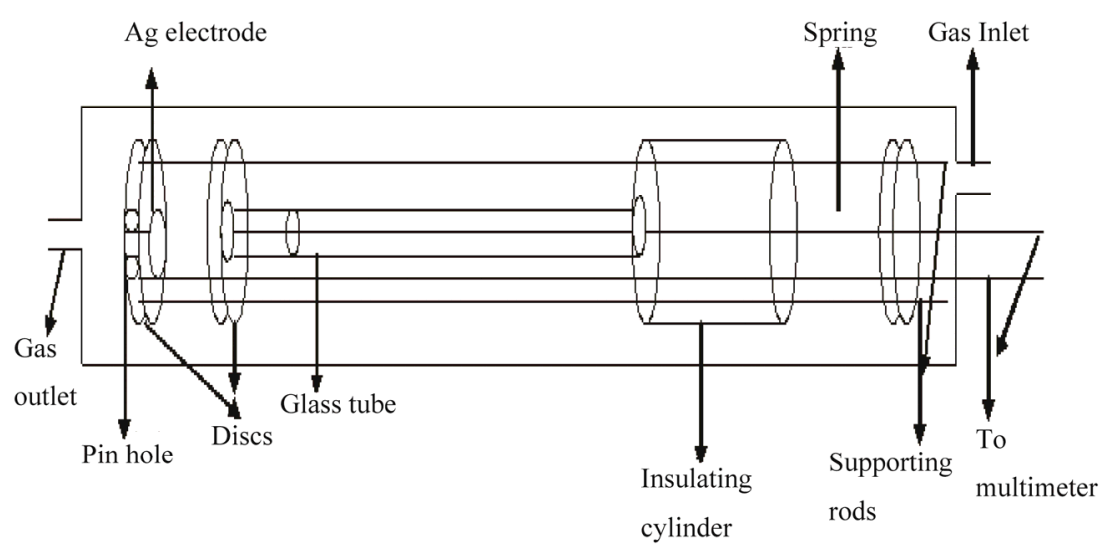

(a)

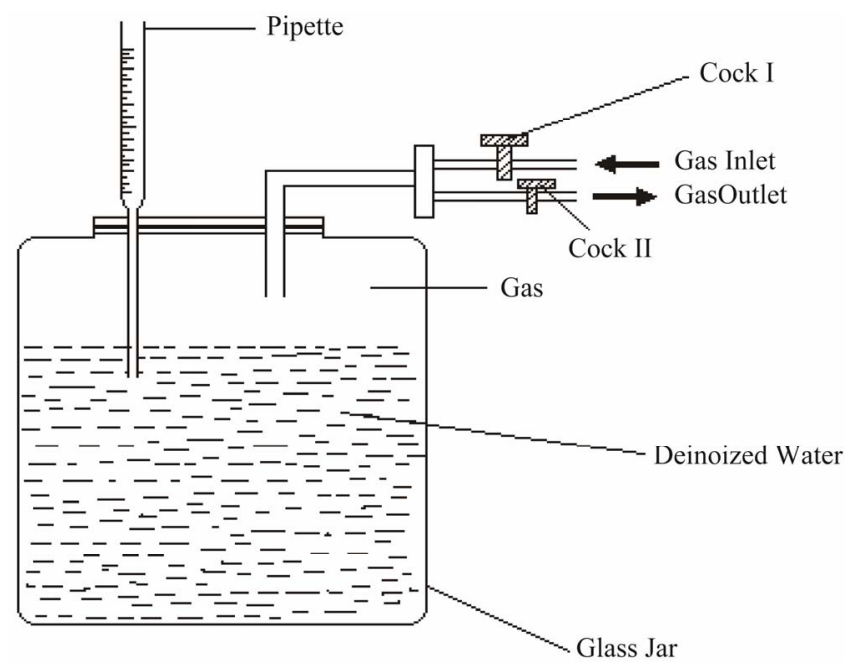

(b)

Figure 4. (a) Device assembly; (b) Concentration measuring system.

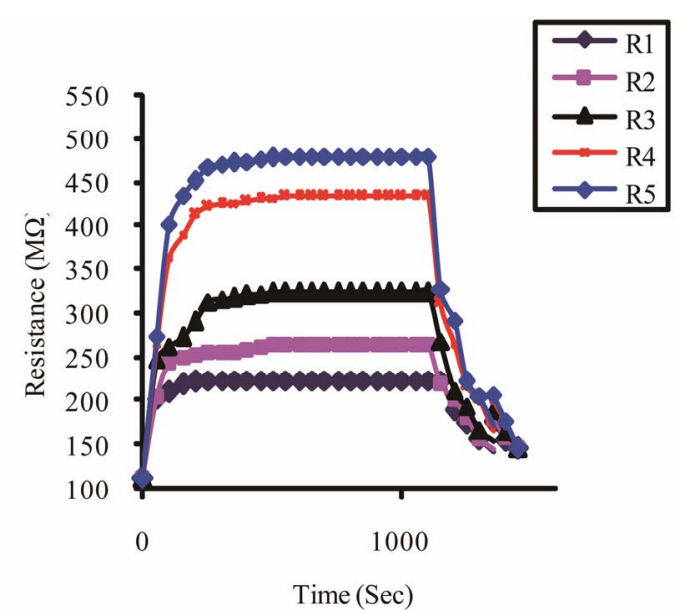

Figure 5. Variation of resistance of $\mathrm{SnO}_{2}$ thick film with the time after exposure for different vol\% of LPG.

surface of the material, which is responsible for the increase in sensitivity of tin oxide thick film. This increase could be attributed to the efficient replenishment of oxy-

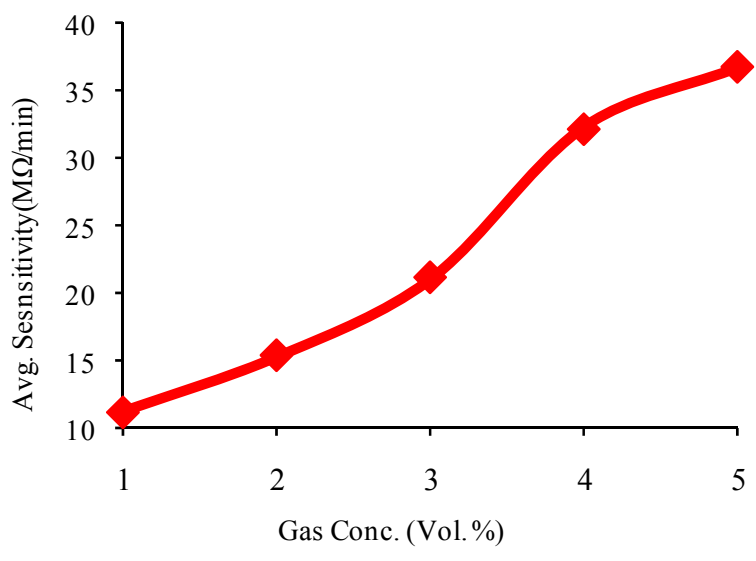

Figure 6. Variations of average sensitivity of sensing film with concentration of LPG.

gen species $\left(\mathrm{O}^{-}, \mathrm{O}^{2-}\right)$ adsorption and desorption process on the tin oxide thick film. Thus, the flow rate of the gas can control the sensitivity of the sample towards LPG. Sensor response curves are shown in Figure 7 manifest that when the LPG was introduced in the gas chamber, 
the sensor response curves drastically increases up to 200 sec for each case then it increases slowly and becomes constant. After $1100 \mathrm{sec}$, when the outlet of chamber was opened sensor response decreases rapidly and it approaches its initial value. The maximum sensor responses were 273 and 312 for 4 and 5 vol\% of LPG. Sensor is reproducible within $\pm 94 \%$ accuracy as shown in Figure 8. The response for film might be recognized to adsorption of LPG and reaction between LPG and the adsorbed oxygen species. The amount of adsorbed oxygen species is fairly important for providing sufficient reactants for the reaction. As the film surface shows more adsorption sites and exposed surface area therefore, it shows better response.

Another important factor for every gas sensor is its response and recovery times, when the sensor is exposed to and then removed from the gas environment. Here, the response time required for the response value to attain $90 \%$ of its maximum value is shorter $(\sim 110 \mathrm{sec})$ for tin

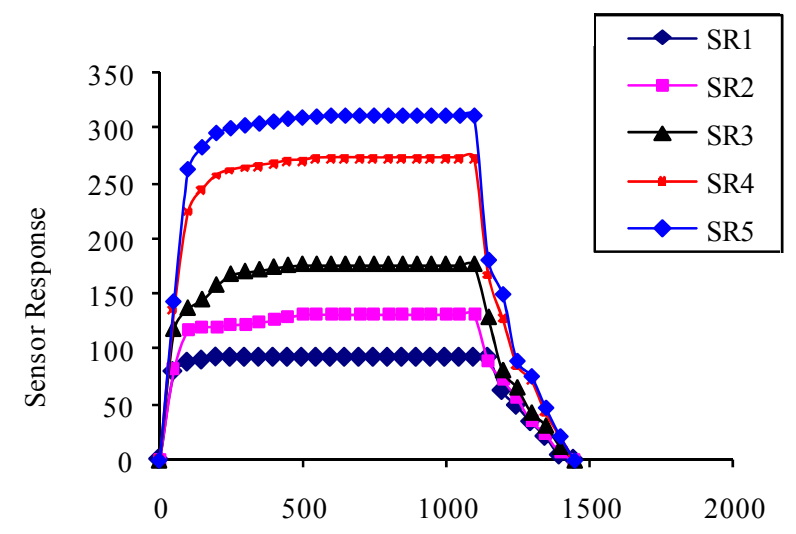

Time (sec)

Figure 7. Sensor response curves of $\mathrm{SnO}_{2}$ thick film with the time after exposure for different vol. \% of LPG.

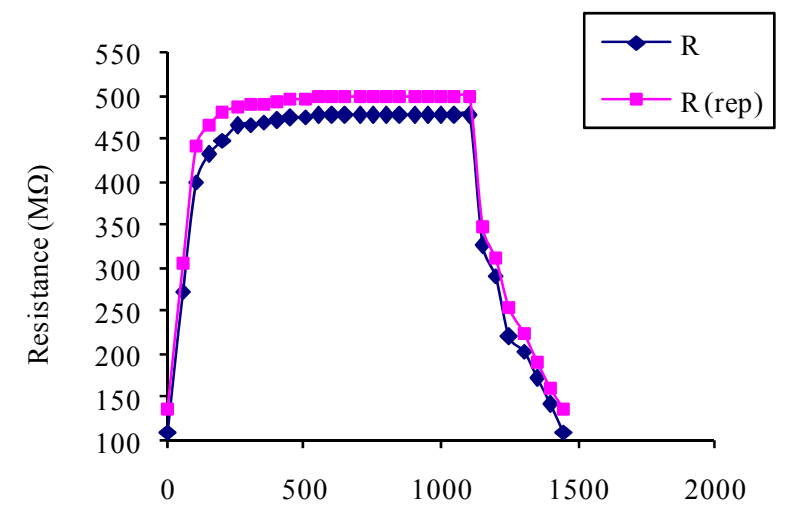

Time (Sec)

Figure 8. Reproducibility curve for the sensing film for 5 vol. \% of LPG. oxide thick film. As the LPG gas was turned-off, the response of the same film fell rapidly, indicating that the good recovery of the resistance was obtained. The time taken by the sensor elements to come back once the LPG was removed is found longer $(\sim 350 \mathrm{sec})$.

It is well known that the sensitivity of the metal-oxide semiconductor sensors is mainly resolute by the interactions between the LPG and the surface of the sensor. So, it is obvious that for the greater specific surface area of the materials, the interaction between the adsorbed gases and the sensor surface will be stronger, i.e. sensitivity will be higher. The gas sensing properties of materials are relative to the surface of the materials. The gases are always adsorbed and even react with the surface. So, small particle size and large specific area contribute to oxygen and LPG adsorption on the surface of the materials, which is responsible for the increase in sensitivity of the sensor.

The LPG sensing mechanism is based on the changes in the resistance of the tin oxide thick film. The oxygen adsorbed on the surface of the film influences the resistance of the tin oxide based sensor. Initially oxygen from the atmosphere adsorbs on the surface of the film and extracts electrons from its conduction bands to form $\mathrm{O}_{2}^{-}$ species on the surface, consequently resistance increases. After that an equilibrium state is achieved between oxygen of $\mathrm{SnO}_{2}$ and atmospheric oxygen and the value is stabilized.

$$
\begin{array}{cc}
\mathrm{O}_{2(\text { gas })} \leftrightarrow \mathrm{O}_{2(\text { ads })} & \text { (film surface) } \\
\mathrm{O}_{2 \text { (gas) }}+\mathrm{e}^{-} \leftrightarrow \mathrm{O}_{2}^{-} & \text {(film surface) }
\end{array}
$$

When the film is exposed to LPG, it reacts with the chemisorbed oxygen. On interaction with hydrocarbons $\left(\mathrm{C}_{\mathrm{n}} \mathrm{H}_{2 \mathrm{n}+2}\right)$ of LPG the adsorbed oxygen is removed, forming gaseous species and water vapor. Consequently, the resistance changes, which is due to the change in the width of depletion layer after exposure to LPG. The overall reaction of LPG with the chemisorbed oxygen may takes place as shown below:

$$
2 \mathrm{C}_{\mathrm{n}} \mathrm{H}_{2 \mathrm{n}+2}+2 \mathrm{O}_{2}^{-} \longrightarrow 2 \mathrm{C}_{\mathrm{n}} \mathrm{H}_{2 \mathrm{n}} \mathrm{O}+2 \mathrm{H}_{2} \mathrm{O}+2 \mathrm{e}^{-}
$$

When the LPG reacts with the surface oxygen ions then the combustion products such as water depart and a potential barrier to charge transport would be developed i.e., this mechanism involves the displacement of adsorbed oxygen species by formation of water. The formation of barrier is due to reduction in the concentration of conduction carriers and thereby, results in an increase in resistance of the sensing element with time. As the pressure of the gas inside the chamber increases, the rate of the formation of such product increases and potential barrier to charge transport becomes strong which has stopped the further formation constituting the resistance 
constant [26-29].

\section{Conclusion}

The morphological and structural investigations have proven the nanocrystalline nature of the tin oxide thick film synthesized through chemical coprecipitation method. The employed chemical route of synthesis was very simple and high yielding. This method can be used for scale-up industrial production of tin oxide. In this way, we may conclude that the detection of Liquid Petroleum Gas is very important for disaster management purpose that's why this study is quite appreciable for commercial applications. It was found that synthesized tin oxide thick film works as a LPG sensor at room temperature. The value of average sensitivity of sensor was $37 \mathrm{M} \Omega / \mathrm{min}$ for $5 \mathrm{vol} \%$ of LPG. The sensor was $94 \%$ reproducible. Thus the experimental results demonstrate that nanosized tin oxide thick film without incorporation of dopants appears to be a promising material for the LPG sensing.

\section{Acknowledgements}

I am highly thankful to Prof. S. K. Omanwar for his encouragement and valuable guidance.

\section{REFERENCES}

[1] H. Suo, F. Wu, Q. Wang, G. Liu, F. Qiu, B. Xu and M. Zhao, "Study on Ethanol Sensitivity of Nanocrystalline $\mathrm{La}_{0.7} \mathrm{Sr}_{0.3} \mathrm{FeO}_{3}$-Based Gas Sensor," Sensors and Actuators B: Chemical, Vol. 45, No. 3, 1997, pp. 245-249. doi:10.1016/S0925-4005(97)00314-6

[2] A. A. Tomchenko, G. P. Harmer, B. T. Marquis and J. W. Allen, "Semiconducting Metal Oxide Sensor Array for the Selective Detection of Combustion Gases," Sensors and Actuators B: Chemical, Vol. 93, No. 1-3, 2003, pp. 126-134. doi:10.1016/S0925-4005(03)00240-5

[3] Y. G. Choi, G. Sakai, K. Shimanoe, Y. Teraoka, N. Miura and N. Yamazoe, "Preparation of Size and Habitcontrolled Nano Crystallites of Tungsten Oxide," Sensors and Actuators B: Chemical, Vol. 93, No. 1-3, 2003, pp. 486-494. doi:10.1016/S0925-4005(03)00195-3

[4] A. B. Bodade, M. Alvi, A. V. Kadu, S. V. Jagtap, S. K. Rithe, P. R. Padole and G. N. Chaudhari, "Synthesis of Nanocrystalline $\mathrm{SnO}_{2}$ Modified $\mathrm{TiO}_{2}$ : A Material for Carbon Monoxide Gas Sensor," Sensors \& Transducers, Vol. 98, No. 11, 2008, pp. 6-15.

[5] N. Yamozoe and N. Mura, "Environmental Gas Sensing," Sensors and Actuators B: Chemical, Vol. 20, No. 2-3, 1994, pp. 95-102. doi:10.1016/0925-4005(93)01183-5

[6] M. N. Rumyantseva, V. V. Kovalenko, A. M. Gaskov, T. Pagnier, D. Machon, J. Arbiol and J. R. Morante, "Nanocomposites $\mathrm{SnO}_{2} / \mathrm{Fe}_{2} \mathrm{O}_{3}$ : Wet Chemical Synthesis and Nanostructure Characterization," Sensors and Actuators B: Chemical, Vol. 109, No. 1, 2005, pp. 64-74. doi:10.1016/j.snb.2005.03.017

[7] H. Tang, K. Prasad, R. Sanjines and F. Levy, "TiO 2 Ana- tase Thin Films as Gas Sensors," Sensors and Actuators B: Chemical, Vol. 26, No. 1-3, 1995, pp. 71-75.

doi:10.1016/0925-4005(94)01559-Z

[8] M. Mabrook and P. Hawkins, "Benzene Sensing Using Thin Films of Titanium Dioxide Operating at Room Temperature," Sensors and Actuators B: Chemical, Vol. 2, No. 9, 2002, pp. 374-382.

[9] J. Q. Xu, Q. Y. Pan, Y. A. Shun and Z. Li, "Emulsion Synthesis Structure and Gas Sensing Properties of Nanometer ZnO," Journal of Inorganic Chemistry, Vol. 14, 1998, pp. 355-359.

[10] P. T. Mosely, "Solid-State Gas Sensors," Measurement Science and Technology, Vol. 8, No. 3, 1997, pp. 223-237. doi:10.1088/0957-0233/8/3/003

[11] A. Srivastava, K. Jain, Rashmi, A. K. Srivastava and S. T. Lakshmikumar, "Study of Structural and Microstructural Properties of $\mathrm{SnO}_{2}$ Powder for LPG and CNG Gas Sensors," Materials Chemistry and Physics, Vol. 97, No. 1, 2006, pp. 85-90. doi:10.1016/j.matchemphys.2005.07.065

[12] U. S. Choi, G. Sakai, K. Shimanoe and N. Yamazoe, "Sensing Properties of $\mathrm{SnO}_{2}-\mathrm{Co}_{3} \mathrm{O}_{4}$ Composites to $\mathrm{CO}$ and $\mathrm{H}_{2}$, , Sensors and Actuators B: Chemical, Vol. 98, No. 2-3, 2004, pp. 166-173. doi:10.1016/j.snb.2003.09.033

[13] D. Kohl, "Surface Process in the Detection of Reducing Gases with $\mathrm{SnO}_{2}$-Based Devices," Sensors and Actuators B: Chemical, Vol. 18, No. 1, 1989, pp. 71-118. doi:10.1016/0250-6874(89)87026-X

[14] J. Gong, Q. Chen, W. Fei and S. Seal, "Micro-Machined Nano-Crystalline $\mathrm{SnO}_{2}$ Chemical Gas Sensors for Electronic Nose," Sensors and Actuators B: Chemical, Vol. 102, No. 1, 2004, pp. 117-125. doi:10.1016/j.snb.2004.02.055

[15] D. Kotsikau, M. Ivanovskaya, D. Orlik and M. Falasconi, "Gas Sensitive Properties of Thin and Thick Film Sensors Based on $\mathrm{Fe}_{2} \mathrm{O}_{3}-\mathrm{SnO}_{2}$ Nanocomposites," Sensors and Actuators B: Chemical, Vol. 101, No. 1-2, 2004, pp. 199206. doi:10.1016/j.snb.2004.02.051

[16] G. Korotcenkov, V. Macsanov, V. Brinzari, V. Tolstoy, J. Schwank, A. Cornet and J. Morante, "Influence of Cu-, Fe-, Co-, and Mn-Oxide Nanoclusters on Sensing Behavior of $\mathrm{SnO}_{2}$ Films," Thin Solid Films, Vol. 467, No. 1-2, 2004, pp. 209-214. doi:10.1016/j.tsf.2004.03.028

[17] R. K. Srivastava, P. Lal, R. Dwivedi and S. K. Srivastava, "Sensing Mechanism in Tin Oxide Based Thick Film Gas Sensors," Sensors and Actuators B: Chemical, Vol. 21, No. 3, 1994, pp. 213-218. doi:10.1016/0925-4005(94)01248-2

[18] P. T. Moseley, "Sensors, New Trends and Future Prospects of Thick- and Thin-Film Gas Sensors," Sensors and Actuators B: Chemical, Vol. 3, No. 3, 1991, pp. 167-174. doi:10.1016/0925-4005(91)80002-2

[19] V. Guidi, M. A. Butturi, M. C. Carotta, B. Cavicchi, M. Ferroni, C. Malagu, G. Martinelli, D. Vincenzi, M. Sacerdoti and M. Zen, "Gas Sensing through Thick Film Technology," Sensors and Actuators B: Chemical, Vol. 84, No. 1, 2002, pp. 72-77. doi:10.1016/S0925-4005(01)01077-2

[20] B. C. Yadav, A. Yadav, T. Shukla and S. Singh, "Experimental Investigations on Solid State Conductivity of Co- 
baltzincate Nanocomposite for Liquefied Petroleum Gas Sensing," Sensor Letters, Vol. 7, No. 6, 2009, pp. 11191123. doi:10.1166/s1.2009.1245

[21] S. D. Bakrania and M. S. Wooldridge, "The Effects of Two Thick Film Deposition Methods on Tin Dioxide Gas Sensor Performance," Sensors, Vol. 9, No. 9, 2009, pp. 6853-6868.

[22] E. Llobet, P. Ivanov, X. Vilanova, J. Brezmes, J. Hubalek, K. Malysz, I. Gràcia, C. Cané and X. Correig, "ScreenPrinted Nanoparticle Tin Oxide Films for High-Yield Sensor Microsystems," Sensors and Actuators B: Chemical, Vol. 96, No. 1-2, 2003, pp. 94-104. doi:10.1016/S0925-4005(03)00491-X

[23] M. R. Vaezi and S. K. Sadrnezhaad, "Gas Sensing Behavior of Nanostructured Sensors Based on Tin Oxide Synthesized with Different Methods," Materials Science and Engineering B, Vol. 140, No. 1-2, 2007, pp. 73-80. doi:10.1016/j.mseb.2007.04.011

[24] R. Savua, M. A. Ponce, E. Joanni, P. R. Bueno, M. Castro, M. Cilense, J. A. Varela and E. Longo, "Grain Size Effect on the Electrical Response of $\mathrm{SnO}_{2}$ Thin and Thick Film Gas Sensors," Materials Research, Vol. 12, No. 1, 2009, pp. 83-87.
[25] B. C. Yadav, R. Srivastava and A. Yadav, "Nanostructured Zinc Oxide Synthesized via Hydroxide route as Liquid Petroleum Gas Sensor," Sensors and Materials Japan, Vol. 21, No. 2, 2009, pp. 87-94.

[26] B. C. Yadav, A. Yadav, S. Singh and T. Shukla, "Experimental Investigations on Nano-Sized Ferric Oxide and Its LPG Sensing," International Journal of Nanoscience, Vol. 10, No. 1, 2010, pp. 135-139. doi:10.1142/S0219581X11007478

[27] B. C. Yadav, R. Srivastava, A. Yadav and V. Srivastava, "LPG Sensing of Nanostructured Zinc Oxide and Zinc Niobate," Sensors Letters, Vol. 6, No. 5, 2008, pp. 714 718. doi:10.1166/sl.2008.m132

[28] T. Shukla, B. C. Yadav and P. Tandon, "Synthesis of Nanostructured Cobalt Titanate and Its Application as Liquefied Petroleum Gas Sensor at Room Temperature," Sensor Letters, Vol. 9, No. 2, 2011, pp. 533-540. doi:10.1166/s1.2011.1508

[29] S. Basu and P. K. Basu, "Nanocrystalline Metal Oxides for Methane Sensors: Role of NobleMetals," Journal of Sensors, 2009, Article ID: 861968. doi: $10.1155 / 2009 / 861968$ 